\title{
Erratum
}

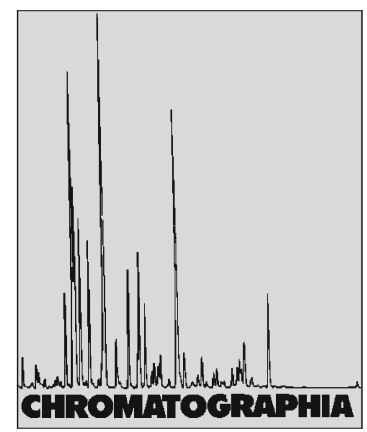

2010, 71, 353

\section{Erratum to: Quantitative Determination of Busulfan in Human Plasma by UPLC}

Quanyun Alan $\mathrm{Xu}^{1, 凶}$, Reza Kazerooni ${ }^{1}$, Jay K. Thapar ${ }^{1}$, Borje S. Andersson ${ }^{2}$, Timothy L. Madden ${ }^{1}$

${ }^{1}$ Department of Experimental Therapeutics, The University of Texas MD Anderson Cancer Center, Houston, TX 77030, USA; E-Mail: qxu@mdanderson.org

2 Department of Stem Cell Transplantation and Cellular Therapy, The University of Texas MD Anderson Cancer Center, Houston, TX 77030, USA

\section{Erratum to: Chromatographia (2009) 70:1505-1510 DOI 10.1365/s10337-009-1334-2}

Unfortunately, in the original article the middle initial of the author Borje S. Andersson was stated incorrectly. The name should read as above. 